\section{Laser photocoagulation of senile macular degeneration}

Senile disciform macular degeneration ${ }^{1-3}$ can now be treated by laser photocoagulation, a therapeutic advance of some importance. This condition is the commonest cause of registered blindness in white communities of the Western world. ${ }^{4}$

In senile disciform macular degeneration blood vessels from the choroid grow between the choroid and retina in the posterior pole of the eye. This process leads to destruction of the pigment epithelium of the retina and serous detachment of the posterior retina. Clinical observations suggest that in the early stages there is rapid growth of new vessels, which tend to grow towards the foveola if they arise at an eccentric site. The growth phase may last a few months and is followed by a stable period, when the complex of new blood vessels remains unchanged. Within two years the subretinal capillaries are usually closed and the retinal detachment has resolved. Visual recovery is rare, however, because there is extensive destruction of tissue, and roughly two thirds of patients are left with a visual acuity of less than $6 / 60$. The cause of the neovascularisation is unknown, but it appears to be the final stage of a series of events at the level of Bruch's membrane (the transparent inner layer of the choroid) which is thought to occur with advancing age. These consist of deposition of debris derived from pigment epithelium of the retina and a reduplication of the basement membrane of the pigment epithelium, which in turn induces an infiltration by macrophages.

Some 14 years ago it was recognised that photocoagulation could be used to destroy the new blood vessels which grow beneath the retina. It was shown that coagulation of a vascular system which did not underlie the foveola could interrupt a disease process that would otherwise progress and cause severe visual loss. Initial studies were undertaken using the xenon arc as an energy source, but results were not encouraging. With the introduction of laser techniques energy could be delivered accurately, through a slit lamp, to small areas of the fundus and so the results improved.

Three randomised controlled trials using laser photocoagulation have recently been carried out in the United States, ${ }^{1}$ France, ${ }^{2}$ and Britain. ${ }^{3}$ These studies were similar in that the patients studied were over 50 and had specific abnormalities on examination-notably drusen (hyaline excrescences in Bruch's membrane) and complexes of new vessels beneath the retina which approached no nearer than $200 \mu \mathrm{m}$ from the foveola in the American study and $100 \mu \mathrm{m}$ in the two European studies. All three studies assessed the potential benefit of argon laser energy using both the green and blue lines available from this laser. The benefits of treatment appeared to be greater in the American study than in the British study. This may be explained by differences between the patients in the two trials; in the American study the patients were generally older with neovascular tissue which was distant from the fovea (these patients derive most benefit from treatment).

Ophthalmologists and their patients should be heartened by the positive results of these prospective randomised clinical trials. Laser photocoagulation, however, is unlikely to be of benefit to many patients unless there is a change in clinical practice. It is well recognised that only a minority of patients with senile disciform macular disease are suitable for treatment when first seen. In most patients the complex of new vessels lies beneath the foveola, and destruction of tissue in this site leads to severe visual loss; it is only the $5-10 \%$ of patients in whom the neovascular complex does not underlie the foveola who will benefit from treatment. Undoubtedly the small proportion of patients with treatable lesions is due to the length of time between the presenting symptom of blurring and distorted central vision and the first visit to an ophthalmologist. In one study $80 \%$ of patients who presented to an ophthalmologist within two weeks of their first symptoms were treatable, but the percentage fell to $40 \%$ when the interval was one month and was less than $10 \%$ when the interval was four months. ${ }^{5}$ Patients with a visual acuity of $6 / 18$ or better were found to have a good chance of having a treatable lesion, whereas those with a visual acuity of $6 / 36$ or worse were unlikely to be suitable for photocoagulation. Hence if patients are seen early in the course of their disease, when the new vessel formation has not reached the foveola, treatment should be successful. The onus falls on primary care physicians to refer their patients for an urgent ophthalmological opinion, a move that in turn has implications, for it will increase the workload of ophthalmologists.

Certain guidelines can be laid down for managing senile disciform macular degeneration in the community. If a patient presents with a short history of visual disturbance and is found to have good visual acuity the likelihood of the causative lesion being treatable is high. Such patients should be fully assessed by carrying out fluorescein angiography as soon as possible. This condition warrants the same degree of urgency as a potential retinal detachment.

Though treatment with argon laser photocoagulation is undoubtedly better than no treatment at all, it is not ideal, since it is relatively ineffective when the neovascular complex is less than $200 \mu \mathrm{m}$ from the foveola. There is reason to believe that the use of the green line of the argon laser alone or the longer wavelength of the krypton laser may increase the number of patients who are potentially treatable and may also improve the results obtained with argon blue and green energy. But there are clearly limitations to this form of treatment, the most important being that the basic disordernamely, the deposition of abnormal material beneath the retina-has not been modified and there is always a risk of recurrent new vessel formation. In addition, there is no evidence that photocoagulation is effective for disciform macular degeneration in any patients except the elderly, and it is also ineffective in those elderly patients with detachment of the pigment epithelium but without subretinal formation of new blood vessels. ${ }^{6}$

A C BIRD

Professor of Clinical Ophthalmology,

Moorfields Eye Hospital,

London ECIV 2PD

${ }^{1}$ Macular Photocoagulation Study Group. Argon laser photocoagulation for senile macular degeneration. Results of a randomized clinical trial. Arch Ophthalmol 1982;100:912-8.

2 Coscas G, Soubrane G. Photocoagulation des neovaisseaux sousretiens dans la degenerescence maculaire senile par le laser a argon: resultats de l'etude randomisee de 60 cas. Bull Soc Ophtalmol Fr (in press).

${ }^{3}$ Moorfields Macular Study Group. Treatment of senile disciform macular degeneration: a single-blind randomised trial by argon laser photocoagulation. Br f Ophthalmol 1982;66:745-53.

4 Sorsby A. The incidence and causes of blindness in England and Wales 1948-62. London: HMSO, 1966:14. (Reports on Public Health and Medical Subjects. No 114.)

${ }^{5}$ Grey RHB, Bird AC, Chisholm IH. Senile disciform macular degeneration; features indicating suitability for photocoagulation. Brf Ophthalmol $1979 ; 63: 85-9$.

${ }^{6}$ Moorfields Macular Study Group. Retinal pigment epithelial detachments in the elderly: a controlled trial of laser photocoagulation. $\mathrm{Br} \mathcal{F} O$ phthalmol $1982 ; 66: 1-16$. 